\title{
Materials Nanoarchitecturing via Cation-Mediated Protein Assembly: Making Limpet Teeth without Mineral
}

\author{
Tina Ukmar-Godec, Luca Bertinetti, John W. C. Dunlop, Aljaž Godec, \\ Michal A. Grabiger, Admir Masic, Huynh Nguyen, Igor Zlotnikov, Paul Zaslansky, \\ and Damien Faivre**
}

Teeth are designed to deliver high forces while withstanding the generated stresses. Aside from isolated mineral-free exception (e.g., marine polychaetes and squids), minerals are thought to be indispensable for tooth-hardening and durability. Here, the unmineralized teeth of the giant keyhole limpet (Megathura crenulata) are shown to attain a stiffness, which is twofold higher than any known organic biogenic structures. In these teeth, protein and chitin fibers establish a stiff compact outer shell enclosing a less compact core. The stiffness and its gradients emerge from a concerted interaction across multiple length-scales: packing of hydrophobic proteins and folding into secondary structures mediated by $\mathrm{Ca}^{2+}$ and $\mathrm{Mg}^{2+}$ together with a strong spatial control in the local fiber orientation. These results integrating nanoindentation, acoustic microscopy, and finite-element modeling for probing the tooth's mechanical properties, spatially resolved small- and wide-angle X-ray scattering for probing the material ordering on the micrometer scale, and energy-dispersive X-ray scattering combined with confocal Raman microscopy to study structural features on the molecular scale, reveal a nanocomposite structure hierarchically assembled to form a versatile damage-tolerant protein-based tooth, with a stiffness similar to mineralized mammalian bone, but without any mineral. microstructure. ${ }^{[2-16]}$ Examples of almost pure mechanical natural selection are found in many different species, used for piercing, biting, cutting, grinding, and/ or scraping. ${ }^{[2-12]}$ Curiously, teeth typically have three fundamental structural components: a hard mineralized working outer layer; a softer supporting and toughening bulk well suited to withstand tension, compression, and impact; and a mechanically graded interface, beneficial for minimizing interfacial stresses. ${ }^{[6]}$ In chiton and limpet teeth, such a strategy gives rise to extreme fracture resistance with tensile strength exceeding $5 \mathrm{GPa} .{ }^{[11]}$ Limpets however exhibit tremendous evolutionary variability as demonstrated by Megatura crenulata, the largest limpet-species that produces teeth completely devoid of mineral. ${ }^{[17]}$ Similar to all molluscs, $M$. crenulata teeth are arranged in rows and anchored to a radula (Figure 1a,b)an elongated flexible tongue-like organ, which continuously regenerates by growing new teeth that replace anterior worn ones, emerging on a conveyor belt-

Biogenic load-bearing structures embody a compromise between biological production cost and animal size constraints and are typically adapted to match a variety of needs. ${ }^{[1-12]}$ Their biomechanical properties usually emerge from contributions across multiple length-scales and gradients in composition and like structure. ${ }^{[5]}$ The teeth are made of $\approx 8 \%$ water, $\approx 60 \%$ chitin, and $\approx 32 \%$ hydrophobic protein rich in Gly $(80 \%)$, His $(10 \%)$, and Pro (5\%) (ref. [17] and Section S1, Supporting Information). These are readily detected by Raman spectroscopy (Section S2, Supporting Information).
Dr. T. Ukmar-Godec, Dr. L. Bertinetti, Dr. J. W. C. Dunlop, M. A. Grabiger, Dr. H. Nguyen, Dr. D. Faivre

Department of Biomaterials

Max Planck Institute of Colloids and Interfaces

Research Campus Golm, 14424 Potsdam, Germany

E-mail: damien.faivre@mpikg.mpg.de

Dr. A. Godec

Mathematical Biophysics Group

Max Planck Institute for Biophysical Chemistry

Am Fassberg 11, 37077 Göttingen, Germany

Prof. A. Masic

Department of Civil and Environmental Engineering

Massachusetts Institute of Technology

Cambridge, MA 02139, USA
Dr. I. Zlotnikov

B CUBE - Center for Molecular Bioengineering

Technische Universität Dresden

01307 Dresden, Germany

Dr. P. Zaslansky

Charite

Julius Wolff Institute for Biomechanics and Musculoskeletal

Regeneration

13353 Berlin, Germany

The ORCID identification number(s) for the author(s) of this article can be found under https://doi.org/10.1002/adma.201701171.

DOI: 10.1002/adma.201701171 

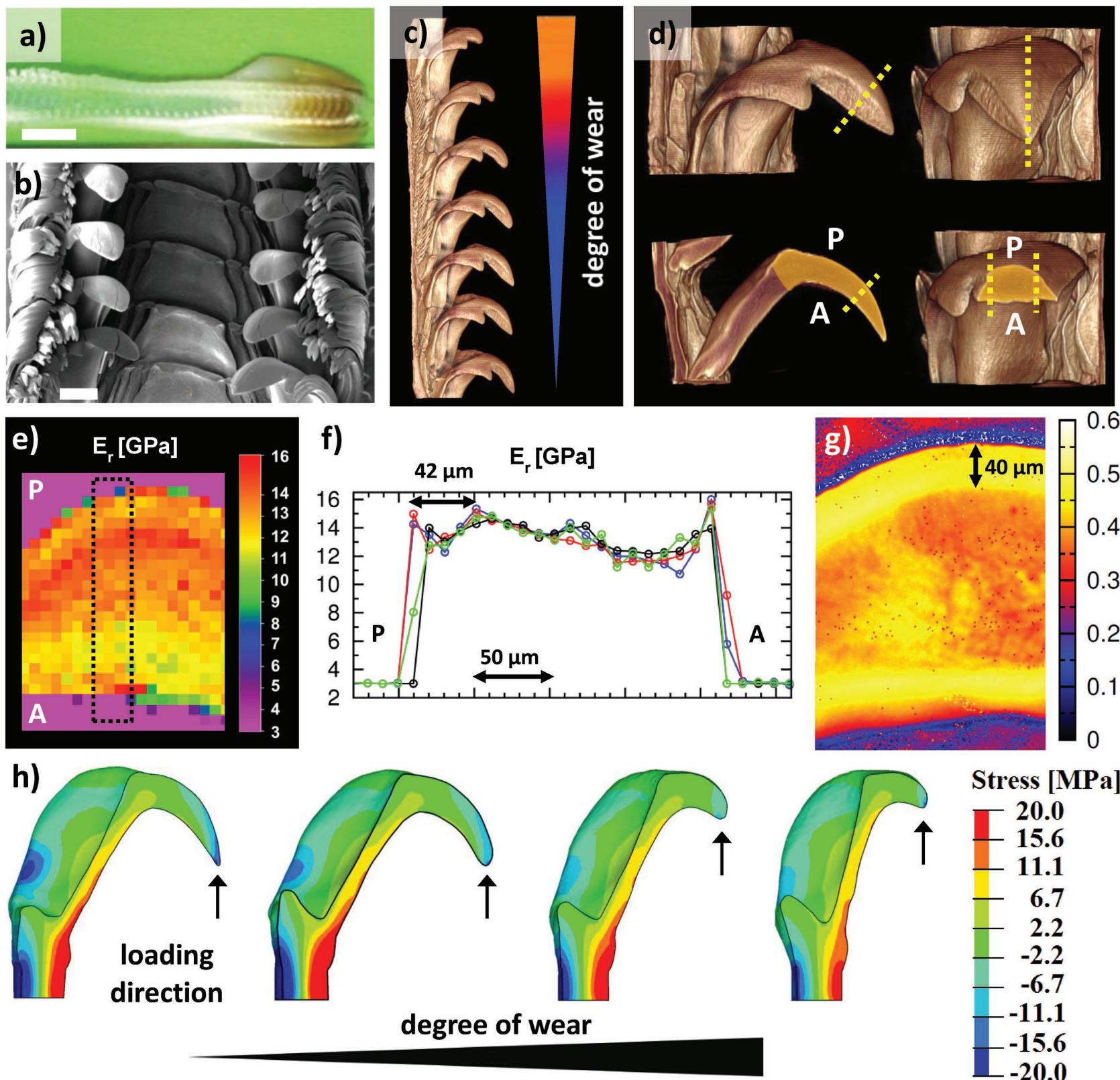

Stress [MPa]

degree of wear

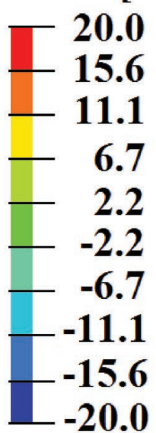

Figure 1. Morphology, wear, and mechanics of teeth. a) An optical image (scale bar $=5 \mathrm{~mm}$ ) and b) Scanning-electron microscopy (SEM) image of the M. crenulata radula (scale bar $=500 \mu \mathrm{m})$; c) Micro-computed tomography $(\mu-\mathrm{CT})$ reconstruction of an array of working teeth depicting progressive signs of wear; d) magnified $\mu$-CT images of a fully formed and operational tooth from (a) depicting two types of sections used for structural and mechanical analysis, longitudinal (left) and transverse (right). P and A denote the posterior (trailing) and anterior (leading) edges of the tooth. e) Maps of reduced modulus on a transverse section depicted by dashed lines in the left panel of (d); f) superimposed adjacent profiles of reduced modulus measured in the area designated in (e); g) scanning acoustic microscopy image depicting the acoustic reflectivity of an area of a transverse section along the plane depicted in bottom right panel of (d). The dashed lines in panel (d) depict the sectioning planes investigated by mechanical testing shown in (e) and (g). h) FEM simulations of longitudinal stresses generated within teeth as a result of applying a force of $125 \mathrm{mN}$ to the tooth tip in the vertical direction (denoted by the arrow). The degree of wear increases toward the right. No significant differences are observed for different stages of wear, demonstrating a versatile wear-tolerant tooth design. The results for three additional loading directions and the corresponding displacement maps are displayed in Section S3 (Supporting Information). Note that the stresses and deformations can depend strongly on the direction of applied force, and M. crenulata teeth are found to be well adapted to different loading scenarios (Section S3, Supporting Information).

The major functioning teeth show progressive signs of wear toward the front, mature end of the radula as evidence of extensive mechanical usage (Figure 1c). Upon emerging from the animal, the complete tooth has the appearance of a sharp knife
(Figure 1b,d). Compared to the iron-oxide mineralizing limpets, its volume is $10-60$ times larger. ${ }^{[17]}$ In order to provide a detailed microstructural and micromechanical understanding of $M$. crenulata mineral-free teeth, we examined longitudinal 
and transverse cross sections (Figure 1d) in a large number of teeth.

Dry nanoindentation experiments reveal a stiffness reaching $16 \mathrm{GPa}$ (Figure 1e,f) when scanning along lines from the edge facing the direction of movement (anterior-A) to the protected posterior $(\mathrm{P})$ edge (see Figure $1 \mathrm{~d}$ ). The stiffness of these teeth appears to compete and even exceed that of heavily mineralized dentin (e.g., in humans, see ref. [18]). Multiple modulus profiles between the $P$ and $A$ tooth edges (Figure $1 \mathrm{f}$ ) reveal well-resolved outer stiffer zones encasing an overall compliant central bulk. A second stiff arch at a distance of about $40 \mu \mathrm{m}$ from the P side (Figure 1f) is also seen, indicative of a multilayer design. When analyzed wet at $92 \%$ relative humidity (RH), stiffnesses of up to $8 \mathrm{GPa}$ were measured in the stiffest part. Profiles obtained in this case reveal the same trend as the dry scans (Section S4, Supporting Information). Hydration thus reduces the stiffness by about half. A higher spatial resolution of mechanical resistance to deformation is seen in impedance maps obtained by scanning acoustic microscopy (SAM, Figure 1g). Measurements with a frequency of $400 \mathrm{MHz}$ and a spatial resolution of $2 \mu \mathrm{m}$ reveal a highly reflective (i.e., stiffer) shell enclosing the less reflective (i.e., more compliant) core (Figure 1g and Section S4, Supporting Information). The width of this reflective shell on the P side is $\approx 40 \mu \mathrm{m}$, which approximately matches the observations obtained from nanoindentation (see also nanoindentation results in hydrated conditions in Section S4, Supporting Information). Such a design juxtaposing compliant and stiff inner and outer layers (Figure 1e,g) is a well-known approach to promote crackdeflection as a damage-resistance mechanism. ${ }^{[4,19]}$ The tooth is thus mechanically designed in the form of an outer compact shell enclosing a more compliant core (Figure 1e-g and Section S4, Supporting Information). The similar distribution of stresses in teeth with different degrees of wear predicted by finite element modeling (FEM) demonstrates that teeth do not lose mechanical integrity upon wear (Figure 1f and Section S3, Supporting Information). Note that similar features are also observed in other, heavily mineralized limpet teeth, typically ascribed to morphology and orientation of goethite particles and chitin fibers. ${ }^{[5,8-10]}$ Yet in $M$. crenulata careful examination has repeatedly demonstrated a complete lack of mineral of any type (see ref. [17] and Section S5, Supporting Information), and hence some other means of reinforcement must be at play.

A better understanding of the tooth architecture on micrometer length-scales emerges from synchrotron-based X-ray scattering, where we measured the degree of relative orientational alignment of chitin fibers (Figure 2a, left panel). Spatial mapping demonstrates highly ordered arcs located roughly $\approx 40 \mu \mathrm{m}$ inward from the A and P edges. On the A-side, the in-plane chitin fiber texture (see Figure 2a, right panel and Figure S6, Supporting Information) corresponds to fibers running parallel to the outer surface. Moving toward the P-edge, the fibers are seen to rotate to an orientation that is perfectly normal to the surface within an $\approx 40-70 \mu \mathrm{m}$ layer near the P-surface. These diffraction measurements additionally confirmed the lack of any mineral phases (see Section S5, Supporting Information).

While crystalline protein domains could possibly explain the higher stiffness zones, ${ }^{[20]}$ the diffraction signals are amorphous suggesting that little if any of the proteins of M. crenulata form crystalline structures (see Section S5, Supporting Information). Interfacial scattering in the high- $q$ Porod regime of the smallangle X-ray scattering (SAXS) domain was used to study protein packing in space. In such measurements, compact phases are delimited by sharp and coherent interfaces, whereas loosely packed assemblies containing empty voids exhibit mass- and/or surface-fractal or even random-coil behavior, depending on how densely matter fills the space (see Section S7, Supporting Information). The results reveal the presence of a densely packed phase in the shell corresponding to thicknesses of $\approx 70$ and $\approx 40 \mu \mathrm{m}$ on the P-and A-edges of the tooth, respectively (see Figure $2 \mathrm{~b}$ and Section S7, Supporting Information). Moving toward the interior core of the tooth the packing transitions to mass-fractal-like and then to a completely random Gaussian-coil-like organization (Figure 2b). Additional hints to this arrangement of the protein are revealed by field-emission gun (FEG) SEM, where differences in the sample surface topography as well as clear radial orientation of chitin fiber-bundles near the P edge are seen (Figure $2 \mathrm{c}$ ).

SEM reveals the $40 \mu \mathrm{m}$ thick shell at the $\mathrm{P}$ edge, below which a gradual transition is seen with brighter spots appearing within a layer located $40-70 \mu \mathrm{m}$ from the edge (Figure $2 \mathrm{c}$ ). The long axis of these elliptic spots, presumably protein-chitin fibrous complexes, shortens toward the tooth core (Figure 2c,d). A closer examination of this region by HR-SEM confirms the transition to circular shape (Figure 2d). These observations are consistent with a gradual rotation of the fibrous complexes and agree with the scattering results in Figure 2a. A gradual increase in the center-to-center distance between fibrous complexes (Figure 2c-e) suggests less dense packing toward the core in agreement with SAXS results (Figure $2 \mathrm{~b}$ ). Moreover, the SEM image of a longitudinal section shown in Figure 2c clearly reveals a distinct $40 \mu \mathrm{m}$ shell along both edges. These findings match the results of nanoindentation and SAM, where a stiffer shell is correlated with higher packing density within the tooth. However, the densely packed region on the P-side is roughly $30 \mu \mathrm{m}$ thicker than the stiffened shell (compare Figure 1e-g with Figure $2 \mathrm{~b}$ ), which requires explanation.

EDX provides further clues into the intricate material assembly revealing a coherent $70 \mu \mathrm{m}$ thick Ca-rich shell encasing a thinner Mg-rich shell located $40 \mu \mathrm{m}$ from the outer tooth surface (see Figure $2 \mathrm{~g}$ and Figure S8, Supporting Information). Curiously, $\mathrm{Cl}$ is not colocalized with these metal ions, suggesting the presence of halogenated residues or $\mathrm{Cl}^{-}$electrostatically bound to positively charged residues in the core (see also Section S8, Supporting Information). The positive charges of $\mathrm{Ca}^{2+}$ and $\mathrm{Mg}^{2+}$ are neutralized by those of acidic residues (Section S1, Supporting Information). The overlap in intensities of the $\mathrm{Ca}$ and $\mathrm{Mg}$ signals and the observed protein packing, as well as chitin organization and mechanical properties, suggests that $\mathrm{Ca}^{2+}$ enhances packing density, whereas $\mathrm{Mg}^{2+}$ increases material stiffness (compare Figure $1 \mathrm{e}-\mathrm{g}$ and Figure $2 \mathrm{~b}-\mathrm{g}$ ). To decipher how this regulation arises we focus on the molecular protein composition and structure.

Raman spectroscopy revealed an overall uniform tooth composition (Figure S2.1, Supporting Information). Nevertheless, non-negative matrix factorization (Sections S2 and S12, Supporting Information) strikingly identified two distinct kinds 
a)

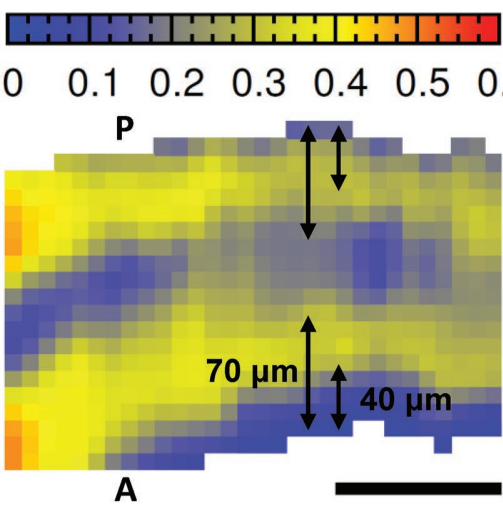

WAXS (chitin fibres)

\section{$\gamma / \gamma_{\max }$} $\mu\left[^{\circ}\right]$

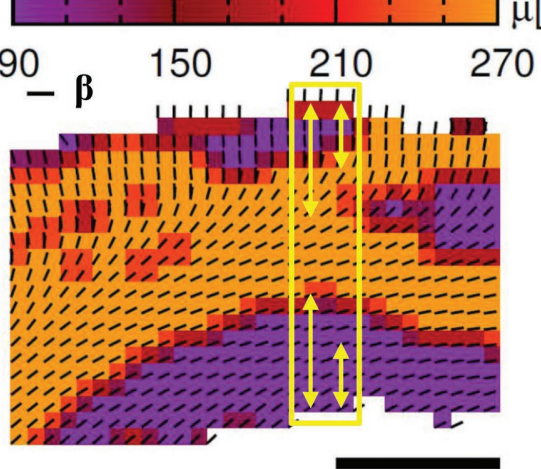

b)

SAXS (proteins)

scattering exponent

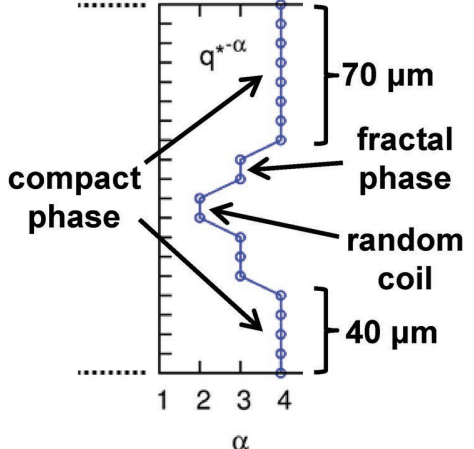

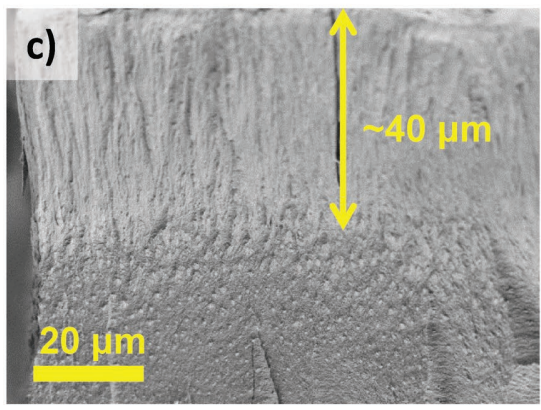
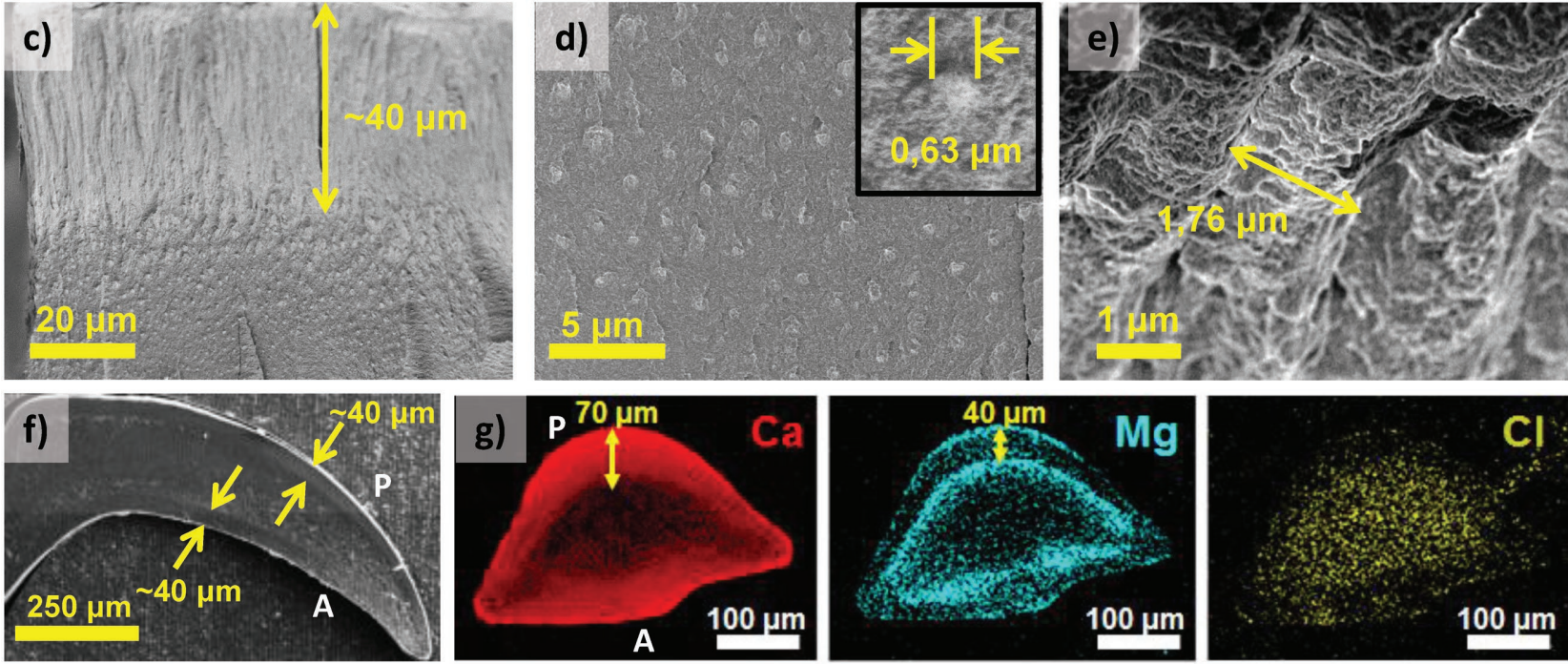

Figure 2. Tooth architectural elements: fiber orientation, protein packing, and cation distribution. a) Wide-angle X-ray scattering (WAXS) revealing (left) the degree of orientational order relative to the maximal order within the sample $\gamma / \gamma_{\max }$ in terms of the ratio of intensities of azimuthal peaks and the isotropic background, $\gamma=I_{\text {peak }} / I_{\mathrm{bgr}}$ and (right) fiber-texture within a longitudinal section of the tooth. In the right panel the color code represents $\mu$, the out-of-plane tilt angle relative to the direction of the beam, and the black lines the in-plane angle $\beta$ of $\alpha$-chitin fibers. The short and long arrows correspond to 40 and $70 \mu \mathrm{m}$, respectively. The scale bar is $100 \mu \mathrm{m}$; b) power-law exponent $\alpha$ in the structurally limited power-law Porod regime (see Section S5, Supporting Information) as a measure for the compactness of the protein assembly ( $\alpha=4$ corresponds to scattering from sharp interfaces delimiting compact phases, $\alpha=3$ to scattering from surface and/or mass fractals and $\alpha=2$ to scattering from random coil-like structures). The results correspond to the area denoted by the yellow rectangle in the right panel of panel (a); c) secondary electron SEM image of a transverse cross section of a broken tooth near the P edge of a tooth depicting a $40 \mu \mathrm{m}$ brighter region gradually turning darker with brighter spots. The entire image height is $70 \mu \mathrm{m}$ corresponding to the $70 \mu \mathrm{m}$ thick region at the P edge in panels (a) and (b). d) Cryo-SEM image of a transverse section distal to the $\mathrm{P}$ edge approaching the core region reveals brighter spots separated by $2 \mu \mathrm{m}$, which presumably correspond to chitin-protein fibrous complexes and are in agreement with the chitin pattern in (a). A closer inspection reveals a decrease in the diameter of these fibrous complexes toward the lower end of the image, suggesting a gradual rotation of the out-of-plane angle in the direction toward the tooth core. Inset: magnification of a fibrous complex in the center of the image. e) Cryo-SEM image of a transverse section in the shell region shows a magnification of the fibrous complexes within the $40 \mu \mathrm{m}$ brighter region near the P-edge depicted in ( $\mathrm{f}$ ). The diameter of these close-packed fibrous complexes (and thereby their center-to-center distance) is $\approx 1.8 \mu \mathrm{m}$. The results therefore reveal a loosening of the packing in the direction of the core; $\mathrm{f}$ ) SEM image of a longitudinal section depicting the $40 \mu \mathrm{m}$ thick shell enclosing a core; g) cross-sectional energy-dispersive X-ray spectroscopy (EDX) elemental maps of $\mathrm{Ca}$ (left), $\mathrm{Mg}$ (middle), and $\mathrm{Cl}$ (right).

of spectra observed within an $\approx 20 \mu \mathrm{m}$ thick shell and the remaining inner core, differing mostly in the chitin bands as a result of a varying fiber orientation (Figure 3a,b and Figure S2.1, Supporting Information). In addition, the fraction of disordered motifs is higher in the core region (see the highlighted bands in Figure 3a and Section S2, Supporting Information). Moreover, shell spectra contain an additional band around $1740 \mathrm{~cm}^{-1}$ (arrow in lower panel of Figure 3a), corresponding to ester groups, which enhance the hydrophobic character of this region. ${ }^{[21]}$

The structural layout of the fiber-reinforced protein composite, depicted schematically in Figure 3c, can be better understood from a materials engineering perspective. The tooth has three fundamental constituents: a hard working part (Figure 3c, green and red shell), a softer supporting part (Figure 3c, blue shell), and a graded interface. The chitin fibers rotate from a 
a)

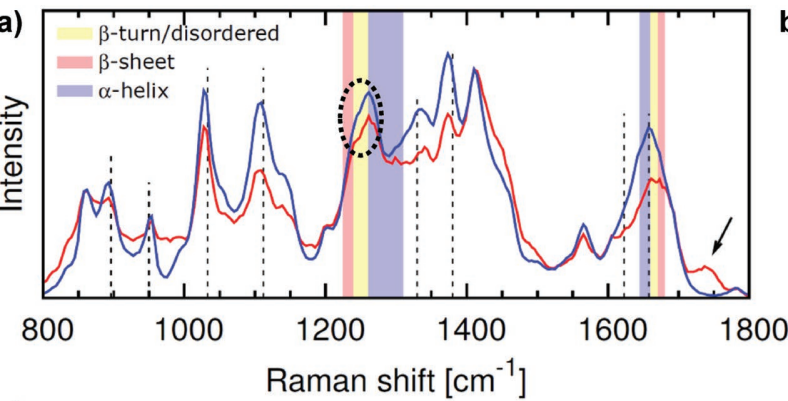

c)

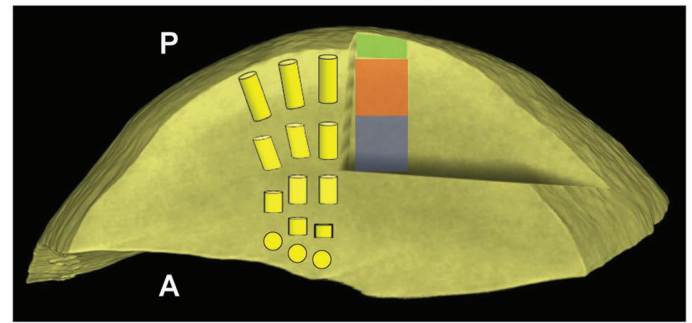

b)
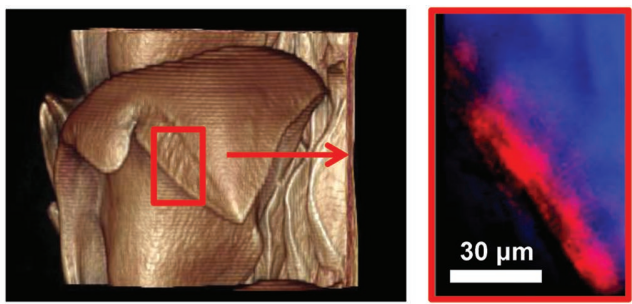

compact protein assembly controlled by $\mathrm{Ca}^{2+}$ and $\mathrm{Mg}^{2+}$

loose, random-coil protein assembly

chitin fibres

Figure 3. Chemical composition and the fundamental design principle of teeth. a) Characteristic Raman spectra of the core (blue line) and shell (red line) region in the tooth obtained with non-negative matrix factorization (NMF). The arrow denotes the peak due to cross-linking esters, which is present only in the surface/shell region; b) (Left) $\mu \mathrm{CT}$ image of the tooth highlighting the region where confocal Raman microscopy (right panel) was performed and the corresponding division into core and shell according to NMF. c) $\mu$-CT reconstruction (left) of a transverse cross section of the tooth with the fundamental architectural elements: The tooth has a compactly packed stiff outer shell, with $\beta$-sheet and $\beta$-turn rich proteins, whose structure is affected by interactions with abundant $\mathrm{Ca}^{2+}$ and $\mathrm{Mg}^{2+}$ ions. In a surface layer (green) the proteins are chemically cross-linked with ester groups providing protection against abrasion. The compliant core is loosely packed with proteins and as such is expected to withstand low tension and compression originating from applying a force to the surface. Chitin fibers (yellow cylinders) gradually rotate from a direction normal to the surface at the P-edge toward lying parallel to the surface on the A-side.

normal orientation to the $\mathrm{P}$ surface to a parallel orientation to the A edge (Figure 3c, yellow cylinders), similar to observations in iron-mineralizing limpets. ${ }^{[5,8,11]}$ More importantly, the chitin texture can be linked to a reinforcement of the mechanical integrity by considering the stresses generated in the tooth. Depending on the orientation of the loading direction, a force applied to the tooth tip can create either tensile or compressive stresses along the A edge, and compressive or tensile stresses along the P edge (see Section S3, Supporting Information). Although exact orientations of teeth loading in vivo are unknown, stress distributions calculated for forces loading perpendicular to the A edge, give rise to slightly higher tensile loading on the A side. Such loading would be equivalent to that produced by a "rasping" action, whereby teeth are dragged along a substrate. In such a situation the tensile load will arise parallel to the local fiber orientation, a more vertical component of force can however reverse the situation (see Section S3, Supporting Information and ref. [10]). In general, damage to the tooth will be guided by the fiber orientation-once failure occurs, the chitin fiber alignment potentially represents "fault lines," which influence the direction of wear thereby preserving the shape and in turn the functionality of the tooth (see Figure 3c). This appears to be analogous to the orientation of magnetite and goethite particles in chiton and limpet teeth. ${ }^{[3,9,10]}$ Moreover, the stiffer shell region does not terminate at a thickness corresponding to the ester cross-linked region (green shell in Figure 3c). Therefore, we propose that, aside from boosting the hydrophobicity, the role of ester cross-linking is analogous to the atacamite reinforcement in Glycera jaws ${ }^{[14]}$ and is most likely designed to enhance abrasion-resistance and not stiffness.

Linking all observations our findings highlight a previously unknown evolutionary strategy of cation-mediated stabilization of hydrophobic protein assembly into mechanically stiff biological tools. The material stiffness therefore integrates effects from the packing of proteins within the chitinous network down to the protein secondary structure, and how these depend on $\mathrm{Ca}^{2+}$ and $\mathrm{Mg}^{2+}$. Gly- and His-rich hydrophobic proteins with extensive $\beta$-sheet motifs, as those observed here, are responsible for the strength of nanofibrillar protein assembly. ${ }^{[22,23]}$ In addition to electrostatic bridging of the sparse acidic residues, $\mathrm{Ca}^{2+}$ and $\mathrm{Mg}^{2+}$ enhance hydrophobic interactions between nonpolar molecules and surfaces ${ }^{[24]}$ thereby promoting their aggregation. Specific $\mathrm{Ca}^{2+}$ binding to peptide carbonyl groups ${ }^{[25,26]}$ induces the formation of compact elastic fibers. ${ }^{[25-27]} \mathrm{Ca}^{2+}$ and $\mathrm{Mg}^{2+}$ exert the opposite effect on polar domains of proteins, by reducing the number of intrabackbone self-interactions and promoting disordered and $\beta$-turn motifs. ${ }^{[22,28,29]}$ Consequently, the conformational entropy increases, rendering the polar parts of polypeptide chain more compliant to deformation. ${ }^{[30]}$ Linking these effects with our observations offers an explanation for why the Ca- and Mg-rich shell region (Figure 3c, red) is both more densely packed and stiffer. The fact that the tooth is stiffest in the Mg-rich region seems to be a consequence of additive stabilization effects of $\mathrm{Ca}^{2+}$ and $\mathrm{Mg}^{2+}$. Corroboratory evidence is obtained from experiments, where the structure is disrupted by removing the cations (see Sections S2.2, S8, and S11, Supporting Information). 
Our results demonstrate that mineral reinforcement is not required to achieve the mechanical stiffness needed for building a tooth. We show that stiffness values as high as those found in mammalian bones and some parts of human teeth, can be obtained by a cunning interplay between cation-controlled material nanostructuring with chitin fibers and protein-secondary structures. There are a few reports of nonmineralized tooth-like structures that are all predominantly protein-based. ${ }^{[13-16,31]}$ In contrast to $M$. crenulata, these other teeth owe their increased mechanical properties to covalent protein cross-linking via aromatic residues and/or by Zn-, $\mathrm{Cu}$-, or Mn-coordinative bonding. ${ }^{[13-16,19]}$ The mechanism discovered in $M$. crenulata is different, and stands in contrast to mineralized teeth, where the overall hardness and stiffness can be traced directly to the properties of mineral inclusions sized tens of nanometers and more. ${ }^{[2-5,15]} M$. crenulata teeth integrate architectural principles found in heavily mineralized teeth but use hydrophobic organic materials to create wear-tolerant shell-core structures with tunable stiffness. The tooth design differs from the architecture of nonmineralized squid beaks, where a spatially heterogeneous cross-linking density gives rise to a large hydration gradient and in turn graded mechanical properties. ${ }^{[15,16]}$ In contrast, $M$. crenulata teeth are chemically homogeneous but display spatial variations in the fiber texture, protein packing, and secondary structure. The design of $M$. crenulata teeth uncovered here could serve as an inspiration for the development of stiff mineral-free materials, for example, by integrating a de novo synthesis and characterization of material composites and multiscale modeling along the lines reported in a recent study. ${ }^{[32]}$ Our results therefore reveal sub-millimeter sized unique biodegradable engineering masterpiece and a spectacular example of convergent tooth evolution.

\section{Supporting Information}

Supporting Information is available from the Wiley Online Library or from the author.

\section{Acknowledgements}

The authors thank J. C. Weaver (Wyss Institute for Biologically Inspired Engineering at Harvard University in Boston) for the initial sample preparation and stimulating discussions of the results. The authors thank Petra Leibner for technical support during mechanical tests and Chenghao Li, Stefan Siegel, and Jens Baumgartner for the help during $X$-ray synchrotron experiments at my-spot beamline. The authors also thank Birgit Schonert for sample embedding preparation, Antje Reinecke for helping with the EDTA samples treatment, Christine PilzAllen for performing SAM measurements, and Carmen Weisskopf for the discussion of SAM results. The authors gratefully acknowledge financial support from Max Planck Society. D.F. acknowledges support from the European Research Council (Starting Grant MB2 No. 256915). P.Z. is grateful for financial support of the German Research Foundation through SPP 1420. A.G. acknowledges financial support in the form of a postdoctoral fellowship from the Alexander von Humboldt Foundation, and the Emmy Noether-programme (GO 2762/1-1) of the German Research Foundation. I.Z. acknowledges financial support provided by Bundesministerium für Bildung und Forschung (BMBF) through grant $03 Z 22 E N 11$. H.N. was financially supported "Image Knowledge
Gestaltung: An Interdisciplinary Laboratory" Cluster of Excellence at the Humboldt University of Berlin through DFG contract EXC1027/1.

\section{Conflict of Interest}

The authors declare no conflict of interest.

\section{Keywords}

limpet teeth, mechanical properties, nanoindentation, radula, structurefunction relationship

Received: February 28, 2017

Revised: April 6, 2017

Published online: May 9, 2017

[1] L. M. Gordon, M. J. Cohen, K. W. MacRenaris, J. D. Pasteris, T. Seda, D. Joester, Science 2015, 347, 746.

[2] J. C. Weaver, Q. Wang, A. Miserez, A. Tantuccio, R. Stromberg, K. N. Bozhilov, P. Maxwell, R. Nay, S. T. Heier, E. DiMasi, D. Kisailus, Mater. Today 2010, 13, 42.

[3] Q. Wang, M. Nemoto, D. Li, J. C. Weaver, B. Weden, J. Stegemeier, K. N. Bozhilov, L. R. Wood, G. W. Milliron, C. S. Kim, E. DiMasi, D. Kisailus, Adv. Funct. Mater. 2013, 23, 2908.

[4] L. K. Grunenfelder, E. E. de Obaldia, Q. Wang, D. Li, B. Weden, C. Salinas, R. Wuhrer, P. Zavattieri, D. Kisailus, Adv. Funct. Mater. 2014, 24, 6093.

[5] D. Faivre, T. U. Godec, Angew. Chem., Int. Ed. 2015, 54, 4728.

[6] S. Bentov, P. Zaslansky, A. Al-Sawalmih, A. Masic, P. Fratzl, A. Sagi, A. Berman, B. Aichmayer, Nat. Commun. 2012, 3, 1.

[7] Y. Ma, S. R. Cohen, L. Addadi, S. Weiner, Adv. Mater. 2008, 20, 1555.

[8] P. van der Wal, J. Ultrastruct. Mol. Struct. Res. 1989, 102, 147.

[9] E. D. Sone, S. Weiner, L. Addadi, Cryst. Growth Des. 2005, 5, 2131.

[10] P. van der Wal, H. J. Giesen, J. J. Videler, Mater. Sci. Eng. C 2000, 7, 129.

[11] A. H. Barber, D. Lu, N. M. Pugno, J. R. Soc. Interface 2015, 12, 20141326

[12] J. D. Currey, J. Exp. Biol. 1999, 202, 3285.

[13] C. C. Broomell, J. R. Soc. Interface 2007, 4, 19.

[14] M. G. Pontin, D. N. Moses, J. H. Waite, F. W. A. Zok, Proc. Natl. Acad. Sci. USA 2007, 104, 13559.

[15] A. Miserez, T. Schneberk, C. Sun, F. W. Zok, J. H. Waite, Science 2008, 319, 1816.

[16] Y. Tan, S. Hoon, P. A. Guerette, W. Wei, A. Ghadban, C. Hao, A. Miserez, J. H. Waite, Nat. Chem. Biol. 2015, 11, 488.

[17] T. Ukmar-Godec, G. Kapun, P. Zaslansky, D. Faivre, J. Struct. Biol. 2015, 192, 392.

[18] P. Zaslansky, Collagen: Structure and Mechanics: Dentin, Springer, New York 2008, Ch. 15

[19] E. Degtyar, M. J. Harrington, Y. Politi, P. Fratzl, Angew. Chem., Int. Ed. 2014, 53, 12026.

[20] B. Crist, Annu. Rev. Mater. Sci. 1995, 25, 295

[21] E. A. Carter, H. G. M. Edwards, Infrared and Raman Spectroscopy of Biological Materials (Practical Spectroscopy): Biological Applications of Raman Spectroscopy (Eds: H. U. Gremlich, B. Yan), Marcel Dekker, New York, 2001, Ch. 11, pp. 421-475.

[22] T. P. Knowles, A. W. Fitzpatrick, S. Meehan, H. R. Mott, M. Vendruscolo, C. M. Dobson, M. E. Welland, Science 2007, 318, 1900. 
[23] V. Latza, P. A. Guerette, D. Ding, S. Amini, A. Kumar, I. Schmidt, S. Keating, N. Oxman, J. C. Weaver, P. Fratzl, A. Miserez, A. Masic, Nat. Commun. 2015, 6, 8313.

[24] R. L. Baldwin, Biophys. J. 1996, 71, 2056.

[25] a) D. W. Urry, T. Ohnishi, Bioinorg. Chem. 1974, 3, 305; b) J. W. DePalma, P. J. Kelleher, L. C. Tavares, M. A. Johnson, J. Phys. Chem. Lett. 2017, 8, 484

[26] N. Matsushima, H. Yoshida, Y. Kumaki, M. Kamiya, T. Tanaka, Y. Izumi, R. H. Kretsinger, Curr. Protein Pept. Sci. 2008, 9, 591.

[27] B. Reinboth, E. Hanssen, E. G. Cleary, M. A. Gibson, J. Biol. Chem. 2002, 277, 3950.
[28] M. G. Cacace, E. M. Landau, J. J. Ramsden, Q. Rev. Biophys. 1997, 30, 241.

[29] K. D. Collins, Methods 2004, 34, 300.

[30] S. Rauscher, S. Baud, M. Miao, F. W. Keeley, R. Pomes, Structure 2006, 14, 1667.

[31] P. A. Guerette, S. Hoon, D. Ding, S. Amini, A. Masic, V. Ravi, B. Venkatesh, J. C. Weaver, A. Miserez, ACS Nano 2014, 8,7170 .

[32] C.-C. Chou, F. J. Martin-Martinez, Z. Quin, P. B. Dennis, M. K. Gupta, R. R. Naik, M. J. Buehler, ACS Nano 2017, 11, 1858. 